\title{
Plataforma con Información Geográfica, de Apoyo al Plan de Evacuación Latacunga, en caso de erupción del Volcán Cotopaxi
}

\section{Platform with Geographic Information, to support Evacuation Plan of Latacunga, in the case of Cotopaxi Volcano eruption}

\author{
Alex Cevallos Culqui \\ Universidad Técnica de Cotopaxi, Departamento de Ingeniería Informática y \\ Sistemas Computacionales, Latacunga, Ecuador. Av. Simón Rodríguez s/n Sector \\ San Felipe. alex.cevallos@utc.edu.ec
}

\section{Gustavo Rodríguez Bárcenas}

Universidad Técnica de Cotopaxi, Departamento de Ingeniería Informática y Sistemas Computacionales, Latacunga, Ecuador. Av. Simón Rodríguez s/n Sector San Felipe.gustavo.rodriguez@utc.edu.ec

\section{Karla Cantuña Flores}

Universidad Técnica de Cotopaxi, Departamento de Ingeniería Informática y Sistemas Computacionales, Latacunga, Ecuador. Av. Simón Rodríguez s/n Sector San Felipe.karla.cantuna@utc.edu.ec

\section{Jorge Rubio Peñaherrera}

Universidad Técnica de Cotopaxi, Departamento de Ingeniería Informática y Sistemas Computacionales, Latacunga, Ecuador. Av. Simón Rodríguez s/n Sector San Felipe. jorge.rubio@utc.edu.ec

\section{RESUMEN}

El 14.VIII.015 a las 04:02, el Volcán Cotopaxi vuelve a despertar, un reporte de descensos de flujos piroclásticos exige la evacuación preventiva en las zonas aledañas, el caos prevalece en ciudades como Latacunga y Sangolquí. Como consecuencia de la falta de socialización de los planes de contingencia, pobladores y rescatistas desconocen zonas de seguridad, albergues, rutas de evacuación, ubicación de personas con discapacidad. El presente estudio busca centralizar y publicar información georreferencial de los planes de contingencia 
ante una posible erupción, para brindar a los pobladores en zonas de riesgo, mejor ubiquidad para la toma de decisiones en su plan de evacuación, por medio de una plataforma web que reciba, calcule y entregue información geográfica de zonas de riesgo, albergues, domicilios, discapacitados, rutas de evacuación. Focalizando los riesgos para el cantón Latacunga, la plataforma administra: Información Geográfica de zonas de riesgo, usando un fichero KML (Keyhole Markup Language) con información geográfica de las zonas afectadas por inundaciones laháricas, la delimitación de estas zonas son el resultado de un modelo matemático para la simulación de flujos laháricos con el software LAHARZ; Información geográfica de albergues, se determina la ubicación georreferencial de los 125 albergues asignados para Latacunga; Información geográfica de personas con discapacidad, se registra geográficamente 84 personas con discapacidad, de la parroquia en zona de riesgo, La Matriz; Determinación de ruta de evacuación, la teoría de geometrías en información geográfica, ha permitido procesar datos geométricos de la superficie terrestre, facilitando el cálculo de distancias con longitudes y latitudes.

Palabras clave: Información geográfica; Cotopaxi; evacuación; discapacidad; lahares; albergues.

\section{ABSTRACT}

On August 14, 2016 at 04:02, Cotopaxi volcano (Quito-Latacunga, Central Ecuador) reawakened, a report of pyroclastic flows requires the preventive evacuation in surrounding areas, so chaos prevails in cities like Latacunga and Sangolqui. As a result of the low socialization of contingency plans, residents and rescuers unknown about security zones, shelters, evacuation routes, location of people with disabilities. The aim of this study is to centralize and to publish geospatial information about contingency plans in the case of a possible eruption, providing to residents in risk areas better ubiquity for decisions making in your evacuation plan. It through a web platform that obtain, calculate and deliver geographic information about risk areas, shelters, users addresses, people with disabilities, evacuations routes. Focusing risks for Latacunga city, the platform manages: Geographic Information of risk areas, for this has been used a KML (Keyhole Markup Language) file with geographical information of the areas affected by lahar floods, the delimitation of these areas are the result of a mathematical model to simulate lahar flows with the software LAHARZ. Geographic Information of shelters, the geo-referenced location of the 125 shelters assigned to Latacunga is determined; Geographic Information of people with disabilities, in the neighborhood "La Matriz" located in risk zone, 84 people with disabilities are registered geographically; Determining evacuation route, the theory of geometries in geographic information has allowed to process geometric data of the earth surface, facilitating the calculation of distances with latitudes and longitudes.

Keywords: geo-referenced; Cotopaxi; evacuation; disabilities; lahar; shelters. 


\section{INTRODUCCIÓN}

Desde una perspectiva global de desastres, las erupciones volcánicas producen relativamente poca mortalidad. Aproximadamente 274443 afectaciones han sido provocadas por erupciones volcánicas según registros históricos documentados, con un estimado de 98386 muertes y 5,6 millones de personas afectadas en el siglo 20. La erupción de 1902 del Monte Pelée en Martinica dio lugar a 30000 muertes, que es el mayor número de víctimas mortales en todo los casos volcánicos del siglo 20. En comparación a desastres, las inundaciones fueron la causa principal de muerte en el siglo 20, lo que resulta en un estimado de 6.8 millones de muertes, el terremoto de Tangshan en 1976 causó 242000 muertes. Los registros históricos muestran que el impacto de las erupciones volcánicas en la población humana está marcada por relativamente pocos eventos catastróficos con largos intervalos entre cada evento (Corominas \& Martí, 2015; Doocy et al., 2013; González-Ferran et al., 2015; Mateos, 2013).

Las erupciones volcánicas ocurren rara vez, existen más de 600 millones de personas que viven en áreas que podrían verse afectados por los peligros volcánicos (Mateos, 2013). Aunque volcanes activos pueden ser una amenaza a las poblaciones que viven alrededor de ellos, también existen elementos que constituyen ventajas tales como suelos fértiles, climas aceptables, así como los medios de subsistencia que se ofrecen a través del turismo, ya que los volcanes pueden ejercer una fuerte atracción (Melo \& Luis, 2013; Moreno, 2015; Ojeda Zújar et al., 2013). Todo ello junto a otros apegos humanos y comunitarios son razones de peso para vivir con los riesgos asociados a los volcanes (Pistolesi et al., 2014; Rodríguez \& Companioni, 2014). La minimización de estos riesgos, por tanto, depende de la comunicación y la colaboración efectiva entre los vulcanólogos, los gestores de riesgos y las comunidades vulnerables (Shroder \& Papale, 2014; Stone et al., 2014; Sword-Daniels et al., 2014)

Los desastres naturales son consecuencia de una variedad de fenómenos de diferente origen, tales como: erupciones volcánicas, terremotos, inundaciones, sequías, deslizamientos, entre otros (Aguilera \& Toulkeridis, 2005). Este tipo de peligros naturales son de mayor probabilidad en zonas que circundan el cinturón de fuego del pacífico, ya que bajo el océano pacífico existen placas tectónicas en constante fricción acumulando energía, que al liberarse, es cuando se da lugar actividades sísmicas o volcánicas (Elberg et al., 2012). Ecuador se encuentra en la zona del cinturón de fuego del pacífico, lo que ha permitido que su cordillera de los andes se dote de peligrosos volcanes, uno de ellos es el volcán Cotopaxi (Barberi et al., 1995).

El 15 de Agosto del 2015, el Instituto Geofísico de la Politécnica Nacional, aproximadamente a las 09:40, reportó el descenso de flujos piroclásticos por el flanco occidental del volcán Cotopaxi, material volcánico que podría causar lahares, indicó el Instituto. Debido a este reporte, la Secretaría Nacional de Gestión de Riesgos declara la evacuación preventiva de los habitantes de las zonas aledañas a las cuencas hidrográficas que nacen en el Cotopaxi (Jara, 2015). Posterior a este informe, el caos se apodera de ciudades como Latacunga y Sangolquí, que junto con la desorganización y la desinformación son los factores que describen el escenario de las calles durante los minutos del suceso. Al finalizar el día, los hechos evidencian en la población, debilidades en sus planes 
de evacuación y conducta, la falta de capacitación e información entre los pobladores, sobre las zonas de seguridad y albergues, propagó el descontrol durante la evacuación de los poblados (Maisanche F. , 2015).

Después de los acontecimientos suscitados y con la alerta amarilla declarada, autoridades nacionales y provinciales refuerzan planes de capacitación y contingencia ante una posible erupción. En las zonas afectadas se realizan capacitaciones masivas y simulacros de evacuación en instituciones: educativas, públicas y privadas (Maisanche F. , 2015); sin embargo por motivos de inaccesibilidad a las capacitaciones y/o movilidad de la población, la confusión persiste entre los pobladores de la zona. Es por esta razón que se ha visto necesario centralizar y publicar determinada información de los planes de capacitación y contingencia, para así facilitar su accesibilidad y uso de acuerdo a la posible movilidad que tenga el poblador de la zona en riesgo (Corominas \& Martí, 2015).

El objetivo de nuestro estudio es centralizar y publicar información geográfica vinculante a los planes de capacitación y contingencia ante una posible erupción del volcán Cotopaxi, para que los pobladores de las ciudades en peligro puedan analizar las zonas de riesgo y albergues más cercanos a su vivienda, así como también registrar la ubicación de pobladores con discapacidad a ser considerados en caso de evacuación. Esto a través de una plataforma web que: entregue información geográfica de las zonas de riesgo, albergues, asignados para el cantón Latacunga, ubicación de personas con discapacidad; y reciba información geográfica de la ubicación domiciliaria del usuario para determinar su mejor ruta de evacuación al albergue más cercano. Para de esta manera brindarle al usuario una mejor ubiquidad para la toma de decisiones en su plan de contingencia ante una posible erupción volcánica.

El caso de estudio se ha focalizado en información de los albergues en zonas seguras que se han asignado al cantón Latacunga y en el registro de información de los pobladores con discapacidad de la parroquia La Matriz. La plataforma web trabaja con cartografía de la ciudad para entregar información geográfica en relación a la ubicación de: albergues, personas discapacitadas, vivienda del usuario, rutas de evacuación.

En las siguientes secciones, primero ilustraremos el área de estudio de la investigación, posteriormente se describe el método adoptado para el cumplimiento de la meta. Finalmente en las dos últimas secciones se reporta los resultados y la discusión de los mismos en relación a las principales implicaciones con respecto al uso de la información de la plataforma web para los planes de contingencia de familias latacungueñas, así como también de los pobladores con discapacidad que se encuentran en zonas de riesgo.

\section{MÉTODO}

\section{Área de estudio}

La última erupción violenta del volcán Cotopaxi se registra en 1877, el coloso está cubierto de nieve con pesados glaciares y se encuentra localizado en 
latitud $00^{\circ} 41^{\prime} 05^{\prime \prime} \mathrm{S}$ y longitud $78^{\circ} 25^{\prime} 54.8^{\prime \prime} \mathrm{W}$ en la región de la cordillera de los Andes, centro del Ecuador, a una distancia de $35 \mathrm{~km}$ al Noreste de Latacunga y de $45 \mathrm{~km}$ al Sureste de Quito. Una erupción violenta afectaría directamente a 800.000 personas que viven en los alrededores del volcán (Andrade et al., 2005).

La historia volcánica acumulada ha permitido determinar que entre los peligros volcánicos más comunes, tenemos: caída de ceniza, emisión de flujos piroclásticos y lahares (fusión del hielo glaciar). De estos, el de mayor peligro y alcance para los pobladores del cantón Latacunga, son los lahares, que recorren las cuencas hidrográficas y en el caso del volcán Cotopaxi considerando los drenajes naturales que se originan en el cono volcánico, se los ha clasificado en tres zonas: zona norte (Sangolquí, San Rafael, Cumbaya, Guayllabamba) con los ríos Pita y El Salto; zona este (La Serena, Puerto Napo, Misahuallí, El Ahuano) con los ríos Tambo y Tamboyacu; zona sur (Lasso, Latacunga, Salcedo) con los ríos Cutuchi, Saquimala y Alaquez (Ordoñez et al., 2013).

El presente estudio se ha focalizado en la zona sur, cantón Latacunga, las extensiones de las zonas que podría ser afectadas por inundaciones de lahares alrededor de los drenajes que nacen en el volcán se han determinado por diversos estudios. Uno de ellos es el resultado de la aplicación de un modelo matemático denominado LAHARZ desarrollado por hidrólogos y vulcanólogos del Servicio Geológico de los Estados Unidos. LAHARZ calcula las superficies planimétricas y transversales de inundación, a lo largo de un drenaje especificado por el usuario, mediante dos relaciones matemáticas que involucran un volumen lahárico hipotético que es proveído como dato de entrada en el programa. Dependiendo del tipo de escenario eruptivo del volcán, la delimitación de las zonas de riesgo podría variar, en la figura 1 se puede apreciar las zonas en riesgo en el caso de un intenso escenario eruptivo (Ordoñez et al., 2013).

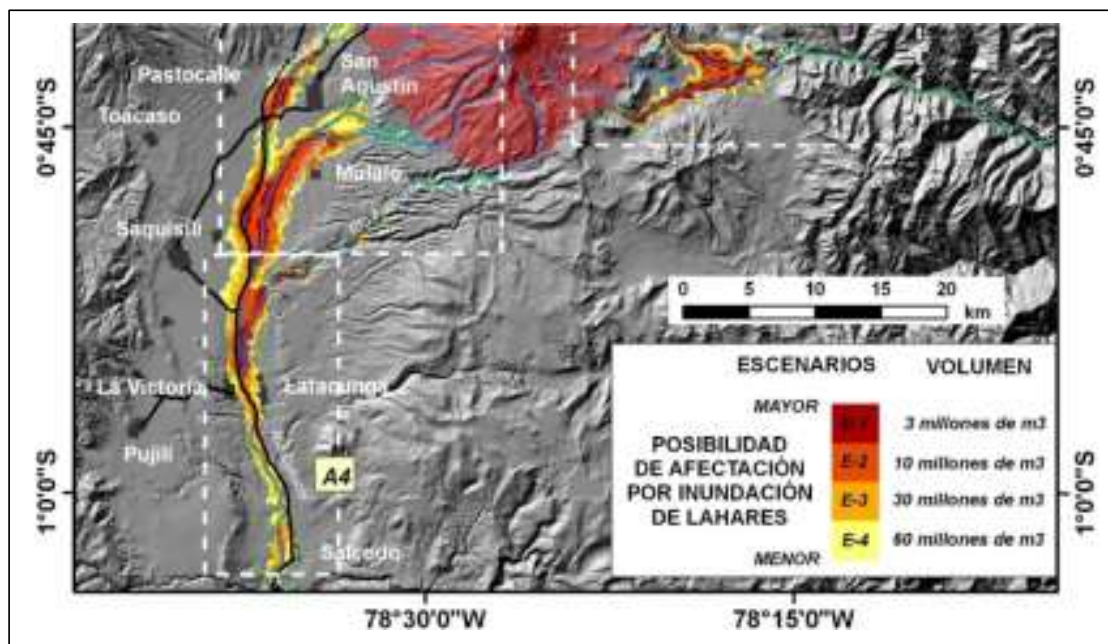

Figura 1 Zonas Sur potencialmente afectadas por inundaciones de lahares. Copyright 2013 por Instituto Geofísico, Las potenciales zonas de inundación por lahares en el volcán Cotopaxi. 


\section{Materiales}

El material base que se ha utilizado para la centralización y publicación de esta información, ha sido el determinado por la Secretaría Nacional de Gestión de Riesgos, que para el caso del cantón Latacunga se han asignado los albergues listados en la Tabla 3. En el caso de los pobladores discapacitados, ha sido información que ha ido el sistema recopilando paulatinamente a través de los registros de los usuarios.

\section{Procedimientos metodológicos}

En el proceso de esta investigación se desarrolla una plataforma web, que acarrea un conjunto de acciones que se muestran a continuación:

1. Determinación de las zonas de riesgo de la ciudad.

2. Ubicación geográfica de los albergues del cantón Latacunga.

3. Determinación de la mejor ruta de evacuación a su albergue más cercano.

4. Registro de datos y ubicación geográfica del poblador discapacitado.

Adicional, para representar el presente desarrollo como un caso de estudio, se han considerado los siguientes aspectos:

- La determinación de aspectos funcionales para el desarrollo del sistema, a través de historias de usuario y la toma de requerimientos.

- Consideración de la arquitectura, diseño e ingeniería en las funcionalidades de cada módulo de la implementación del sistema.

- Pruebas de funcionalidad y aceptación de cada uno de los módulos que conforman el sistema.

Considerando la visión del caso de estudio, la apliación es estructurada en cuatro módulos de trabajo que se describen en la figura 2.

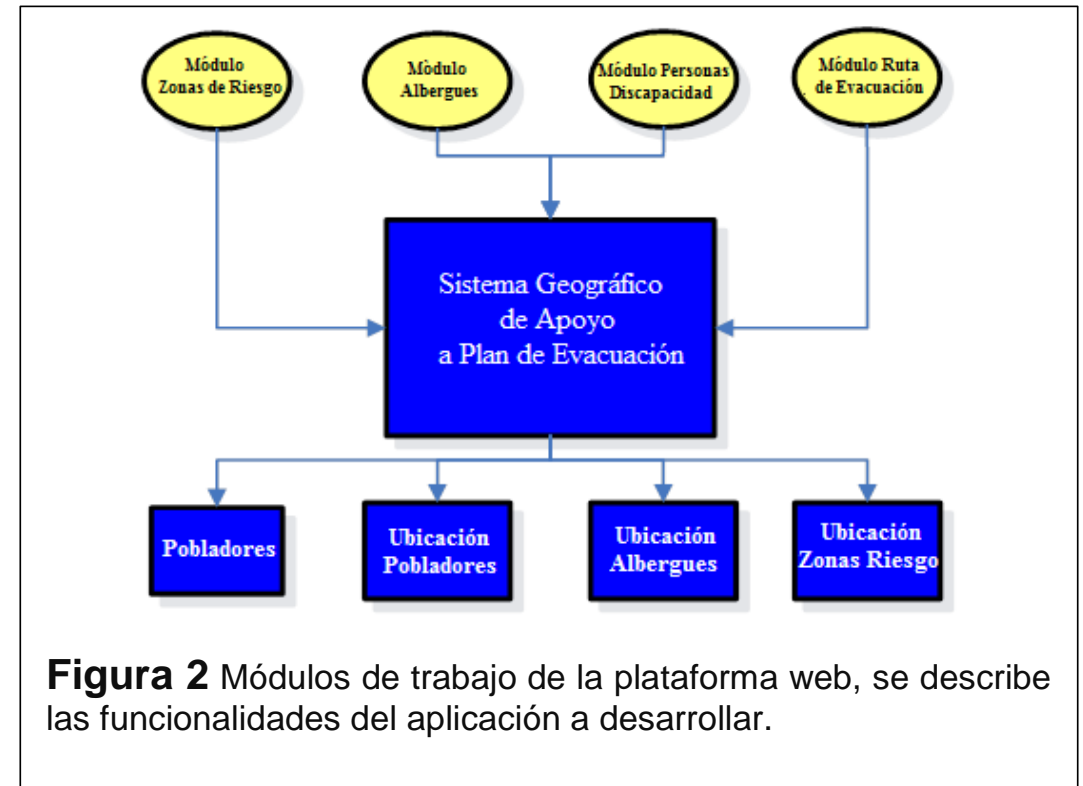


Módulo de Zonas de Riesgo: Delimita las areas geográficas con mayor riesgo de ser afectadas por flujos laháricos.

Módulo de Albergues: Muestra la ubicación geográfica de los 125 albergues asignados a Latacunga por la Secretaría Nacional de Gestión de Riesgos.

Módulo de Personas con Discapacidad: Permite el registro geo-referencial de usuarios, en el caso de un poblador con discapacidad la aplicación muestra su ubicación con la identificación que corresponde.

Módulo de Ruta de Evacuación: De acuerdo a la ubicación geográfica del usuario, la aplicación le presenta la ruta de evacuación al albergue más cercano.

Todos los módulos del entorno de trabajo del caso de estudio implican interacción con cartografía, pues de esta manera se ofrece al usuario un mejor análisis ante la posibilidad de ocurrencia del fenómeno natural. Para sortear esta necesidad se hace uso de la Google Maps Javascript API ${ }^{1}$ herramienta tecnológica que facilita la integración de mapas en un sitio web, con esta base estructural es como se desarrollan los servicios propuestos en este caso de estudio.

\section{1) Módulo de Zonas de Riesgo:}

Para el desarrollo de este módulo ha sido necesario la generación de un archivo que permita el almacenamiento de datos geográficos, este archivo ha sido generado con el programa LAHARZ, que por medio de un modelo matemático calcula el nivel de inundación en una superficie a lo largo de un drenaje, en este caso la fuente a considerar ha sido el río Cutuchi. Es importante mencionar que el programa LAHARZ trabaja con un dato de entrada que es el volumen laharico hipotético, el cual define el tipo de escenario eruptivo, desde un nivel menor a un nivel mayor, el mapa presentado posee la gráfica de un posible escenario eruptivo de nivel mayor.

El mapa ha sido extraído a un archivo de extensión .KML permitiendo ser incorporado como una capa más en entornos cartográficos, en la figura 3 se puede apreciar el archivo cargado en Google Earth. Para que este archivo pueda ser usado en la aplicación, se importa el archivo .KML a la Google Maps API, así se crea un nuevo mapa que es visualizado en la interfaz de la aplicación a través de un iframe ${ }^{2}$. De este modo es como se delimitó las zonas de riesgo y las zonas de seguridad del cantón Latacunga, para este caso de estudio.

\footnotetext{
${ }^{1}$ Aplicación de interfaz de programación para integración de mapas de GooleMaps

${ }^{2}$ Es un objeto HTML embebido en otro documento HTML.
} 


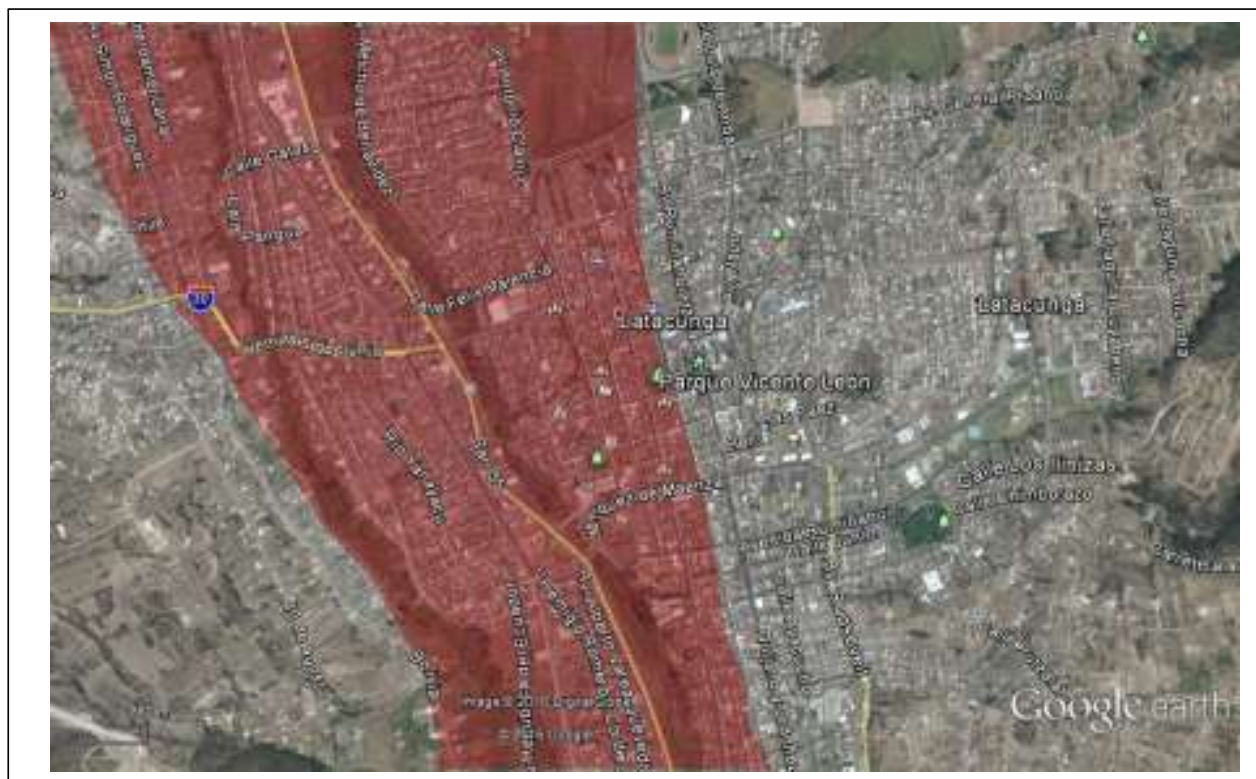

Figura 3 Archivo KML con información geoespacial para ser cargada como una capa más en GoogleEarth.

\section{2) Módulo de Albergues}

Para el registro de la ubicación geográfica de todos los albergues asignados para el cantón de Latacunga, se ha obtenido información de la Secretaría Nacional de Gestión de Riesgos, para lo cual se ha considerado únicamente los registros de las parroquias en zonas de riesgo del cantón en estudio. En la Tabla 1 se puede apreciar el número de albergues por parroquia que se han identificado.

\section{Tabla 1}

Número de albergues por parroquia del Cantón Latacunga

\begin{tabular}{cc}
\hline Parroquia & Número Albergues \\
\hline 11 de Noviembre & 2 \\
\hline Alaquez & 9 \\
\hline Belisario Quevedo & 4 \\
\hline San Buenaventura & 3 \\
\hline Chantilin & 6 \\
\hline Eloy Alfaro & 42 \\
\hline Joseguango Bajo & 2 \\
\hline Juan Montalvo & 17 \\
\hline La Matriz & 13 \\
\hline Mulalo & 19 \\
\hline Tanicuchi & 1 \\
\hline Zumbalica & 7
\end{tabular}


Las coordenadas receptadas de los albergues poseen un formato UTM ${ }^{3}$. Por esta razón ha sido necesario convertirlas a un formato tradicional de coordenadas latitud y longitud, ya que la Google Maps API requiere este formato para operar coordenadas geo-referenciales.

Determinada la ubicación geográfica de los albergues el siguiente paso ha sido publicarlos en cartografía. Para la publicación de estos puntos de ubicación, primero se almacenó las coordenadas de latitud y longitud en un motor de base de datos y para que sean visualizados en la cartografía de GoogleMaps se utilizó la Clase de Superposición Marker ${ }^{4}$ de esta manera se logra publicar las coordenadas geo-referenciales de todos los albergues

\section{3) Módulo de Personas con Discapacidad}

Inicialmente se desarrolla en la aplicación un ambiente para que el usuario pueda registrar una ubicación geográfica que correspondería a su domicilio, conjuntamente con información complementaria a su identificación y condición de discapacidad, esta última en caso de poseerla. En esta ocasión las coordenadas de latitud y longitud las entrega el usuario a través de su interacción con la cartografía de la interfaz de la aplicación. El usuario a través de un "Drag and Drop" 5 pueda modificar la posición geográfica de un marker hasta precisar cuál es la ubicación de su domicilio, para esto, ha sido necesario la utilización de un receptor de eventos de JavaScript denominado addListener(). Es así como se retiene la longitud y latitud que el usuario elija, para posteriormente guardarlo en la base de datos.

En una segunda instancia, se desarrolla en la aplicación una interfaz donde se recopila la ubicación geográfica de todos aquellos usuarios registrados que poseen alguna discapacidad. De igual manera que el módulo dos, para la publicación de estas coordenadas geo-referenciales se utilizó la Clase de Superposición Marker. Esta funcionalidad apoya al plan de las banderas blancas fomentado por las autoridades de las localidades en zonas de riesgo volcánico. En la Tabla 2, se muestran los tipos de discapacidad que se han detectado en el transcurso de esta investigación.

Tabla 2

Tipos de discapacidad identificados

\begin{tabular}{ll}
\hline Discapacidad & Tipo de Discapacidad \\
\hline Retraso Mental & Conductual Mental \\
\hline Visual & Sensorial y de la comunicación \\
\hline Auditiva & Sensorial y de la comunicación \\
\hline Intelectual & Conductual Mental \\
\hline Lenguaje & Sensorial y de la comunicación \\
\hline Física & Motricidad \\
\hline Auditiva & Sensorial y de la comunicación \\
\hline
\end{tabular}

\footnotetext{
${ }^{3}$ Sistema de coordenadas universal transversal de Mercator

${ }^{4}$ Clase que permite la construcción de objetos relacionados con coordenadas de latitud y longitud sobre un mapa

${ }^{5}$ Expresión informática que se refiere a la acción de mover con el cursor un objeto de la interfaz a otra ubicación
} 


\section{4) Módulo de Ruta de Evacuación}

Para (Olaya, 2014) "una de las características del dato espacial deriva de su posición. Esta posición se entiende en términos absolutos (posición de una entidad en el espacio expresada por sus coordenadas) como relativos (relación con otras entidades también en dicho espacio)". Entre los factores elementales del componente espacial se deriva la localización del objeto es decir la posición del objeto en el espacio y las relaciones espaciales en las que difieren las relaciones topológicas y las relaciones geométricas. Las relaciones geométricas son cuantitativas y se obtienen a partir de las coordenadas de los objetos mediante cálculos espaciales básicos tanto en el plano como en el espacio (Pastor \& Pérez, 2008).

La mayor parte de los análisis espaciales hacen uso de la teoría de geometrías la cual incluye operaciones geométricas sencillas a partir de las cuales se generan algoritmos complejos que facilitan el cálculo de datos geométricos de la superficie terrestre, como el cálculo de distancia, el rumbo y el área a partir de coordenadas esféricas (latitud y longitud).

En consecuencia, el módulo de ruta de evacuación hace uso de la teoría de geometrías explícitamente de la geometría esférica a través de la Google Maps API que utiliza una esfera para escenificar la tierra y la proyección 2D para representarla en una superficie bidimensional. El cálculo de la menor distancia entre dos puntos de una esfera no es una línea recta es un segmento de un círculo máximo o línea geodésica y los ángulos que componen un triángulo en la superficie de la esfera suman más de 180 grados. De ahí que Google Maps API emplea la función computeDistanceBetween() que calcula la distancia entre dos coordenadas esféricas, en efecto este cálculo se basa en la fórmula de Haversine (ver expresión 1).

$$
d=2 r \arcsin \left(\sqrt{\sin ^{2}\left(\frac{\emptyset_{2}-\emptyset_{1}}{2}\right)+\cos \left(\emptyset_{1}\right) \cos \left(\emptyset_{2}\right) \sin ^{2}\left(\frac{\delta_{2}-\delta_{1}}{2}\right)}\right)
$$

Donde $\emptyset_{1}, \emptyset_{2}, \delta_{1}, \delta_{2}$ representan la latitud y la longitud expresada en radianes de los puntos 1 y 2 respectivamente, $r$ corresponde al radio terrestre que puede ser ecuatorial $=6378.1 \mathrm{~km}$, polar $=6356.8 \mathrm{~km}$ y medio $=6371.0 \mathrm{~km}$ (Sinnott, 1984). En síntesis este módulo informa a los ciudadanos latacungueños en zonas de influencia del volcán Cotopaxi el camino que deben transitar para evacuar en el menor tiempo posible hacia el albergue más cercano para proteger su integridad.

\section{RESULTADOS}

Los resultados del caso de estudio planteado han sido obtenidos considerando la realidad y la situación de riesgo volcánico del cantón Latacunga. Los datos de ubicación geográfica y zonas de riesgo, necesarios para el desarrollo de la aplicación, ha sido información extraída de: la Secretaría Nacional de Gestión de Riesgos, el software Laharz e información que los pobladores de la parroquia La Matriz han entregado.

Desarrollada y validada la aplicación, en la figura 4 se muestra las secciones de la interfaz principal obtenida, resultado de la cohesión de los cuatro módulos planificados y estructurados. 


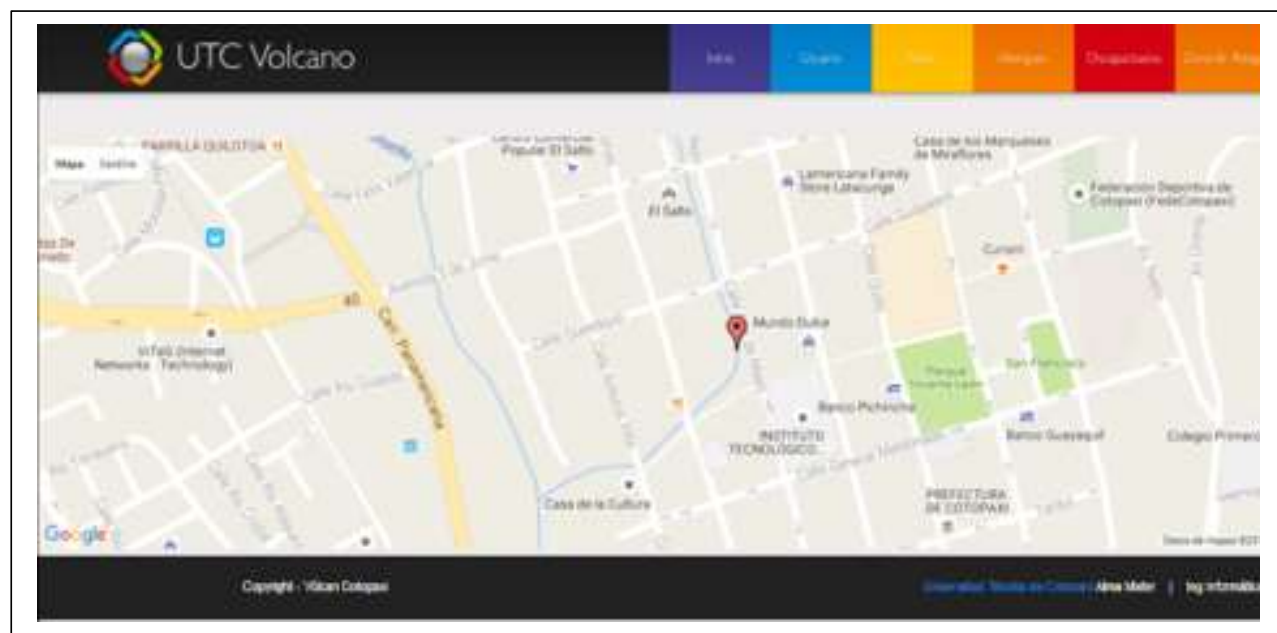

Figura 4 Secciones de la interfaz principal: sección de cabecera, sección de cartografía, sección pie de página.

1) Módulo de Zonas de Riesgo

La integración del archivo .KML con la Google Maps API, ha permitido obtener la delimitación de zonas de riesgo del cantón Latacunga. En la figura 5 se puede apreciar, que en la zona norte los confines de riesgo se encuentran entre la Av. Rumiñahui (Estadio La Cocha) al Este y la Av. 10 de Agosto (Iglesia San Felipe) al Oeste. $Y$ en el sur los confines de riesgo se encuentran entre la Av. Bolivia (Planta Holcim Latacunga) al Oeste y la Av. Quijano y Ordoñez (Colegio de Médicos) al Este.

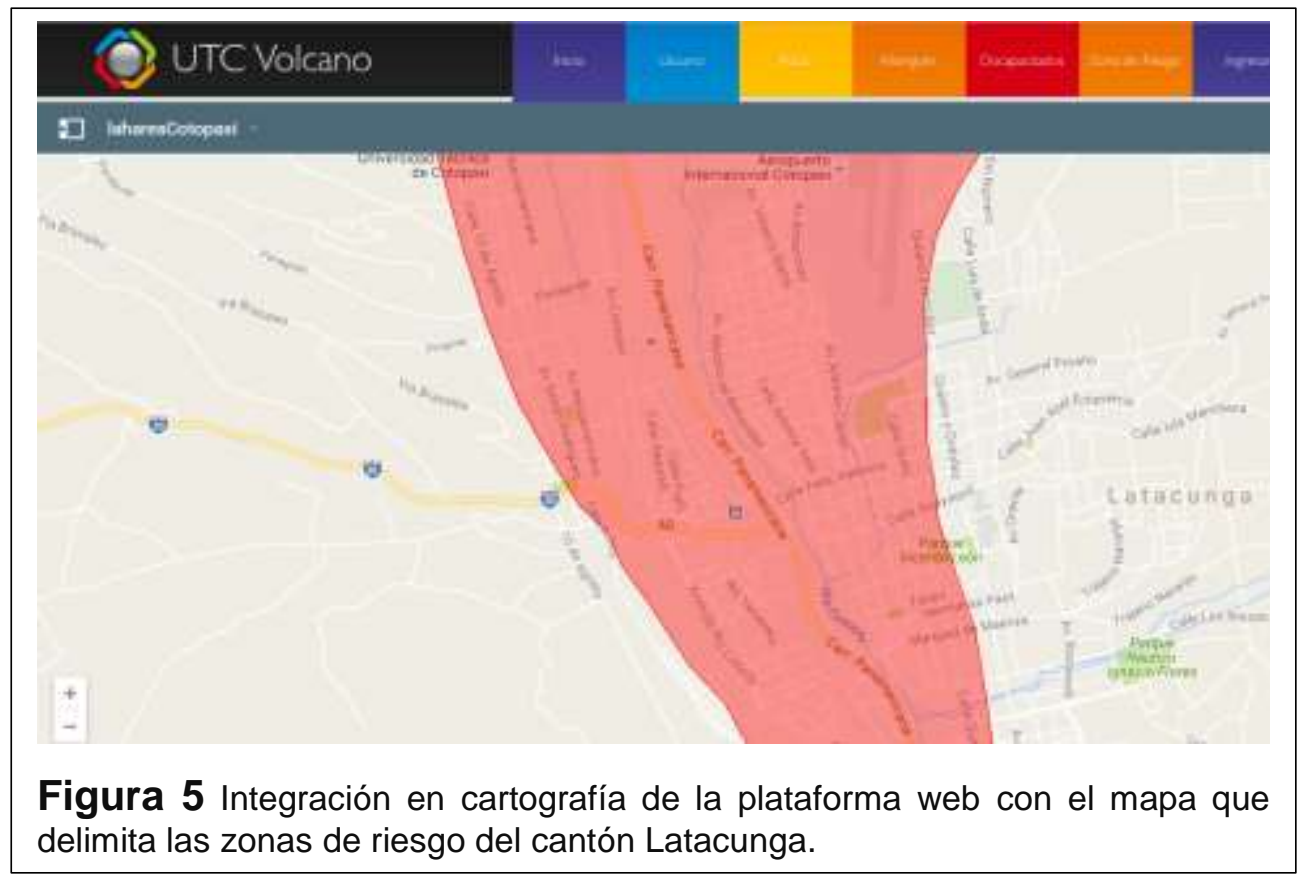




\section{2) Módulo de Albergues}

Permite identificar la ubicación geográfica de los albergues asignados al cantón de Latacunga por parroquia. La información recopilada de los albergues y los datos geo-referenciales en formato original (UTM) y formato Latitud Longitud se los puede apreciar en la Tabla 3. Por motivos de espacio no se presenta todos los registros de los albergues, a modo de ejemplo se presenta algunos de ellos.

\section{Tabla 3}

Coordenadas geográficas de albergue del cantón Latacunga

\begin{tabular}{|c|c|c|c|c|c|c|c|}
\hline \multirow[t]{2}{*}{ № } & \multirow[t]{2}{*}{ Parroquia } & \multirow[t]{2}{*}{ Comunidad } & \multirow[t]{2}{*}{ Establecimiento } & \multicolumn{2}{|c|}{$\begin{array}{c}\text { Coordenadas } \\
\text { UTM }\end{array}$} & \multicolumn{2}{|c|}{$\begin{array}{l}\text { Coordenadas } \\
\text { Tradicional }\end{array}$} \\
\hline & & & & Este & Norte & Longitud & Latitud \\
\hline 1 & $\begin{array}{c}11 \mathrm{de} \\
\text { noviembre }\end{array}$ & $\begin{array}{l}\text { Barrio las } \\
\text { parcelas }\end{array}$ & Casa barrial & 76008 & 98997 & -0.9063 & -78.6630 \\
\hline 2 & $\begin{array}{c}11 \mathrm{de} \\
\text { noviembre }\end{array}$ & $\begin{array}{l}\text { Barrio las } \\
\text { parcelas }\end{array}$ & Coliseo & 76008 & 98997 & -0.9063 & -78.6630 \\
\hline 3 & $\begin{array}{c}\text { Juan } \\
\text { Montalvo }\end{array}$ & $\begin{array}{l}\text { Barrio el } \\
\text { calvario }\end{array}$ & Casa Barrial & 76575 & 98968 & -0.9327 & -78.6121 \\
\hline .. & .. &.. & &.. &.. & .. &.. \\
\hline 122 & Mulalo & Trompucho & $\begin{array}{l}\text { Casa Barrial } \\
\text { Trompucho }\end{array}$ & 77149 & 99121 & -0.7942 & -78.5606 \\
\hline 123 & Mulalo & $\begin{array}{l}\text { Macalo } \\
\text { grande }\end{array}$ & $\begin{array}{c}\text { Hacienda San } \\
\text { Elias }\end{array}$ & 77085 & 99126 & -0.7898 & -78.5663 \\
\hline 124 & $\begin{array}{c}\text { Joseguango } \\
\text { bajo }\end{array}$ & $\begin{array}{c}\text { Centro } \\
\text { parroquial }\end{array}$ & Cibv & 76863 & 99114 & -0.8007 & -78.5863 \\
\hline 125 & $\begin{array}{c}\text { Joseguango } \\
\text { bajo }\end{array}$ & $\begin{array}{c}\text { Centro } \\
\text { parroquial }\end{array}$ & Casa barrial & 76862 & 99113 & -0.8014 & -78.5864 \\
\hline
\end{tabular}

Se ha considerado los 125 albergues asignados por la Secretaria de Gestión de Riesgo para el cantón de Latacunga, obtenidas las coordenadas de ubicación geográfica de cada uno de ellos, estas son almacenadas en base de datos y publicadas en cartografía, como muestra la figura 6.

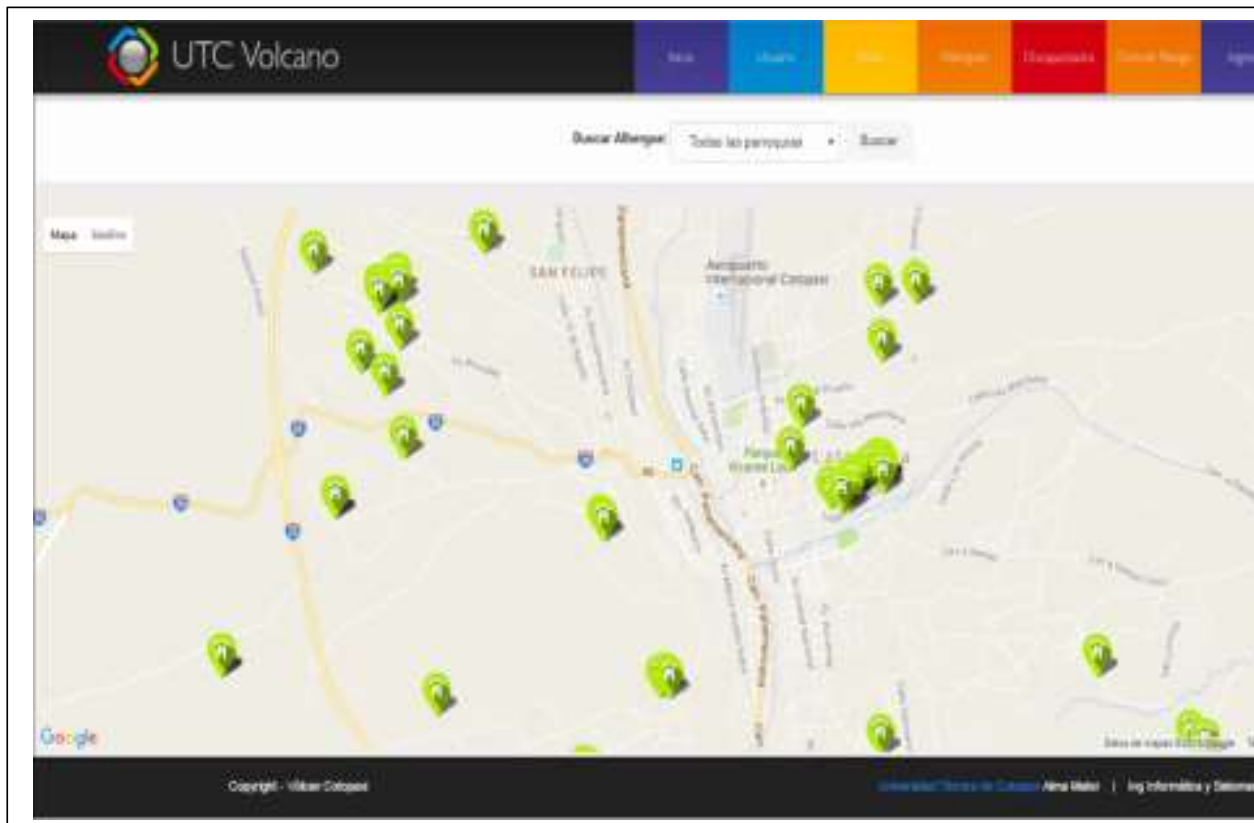

Figura 6 Ubicación geo-referencial en la plataforma web de los 125 albergues asignados para el cantón de Latacunga. 


\section{3) Módulo de Personas con Discapacidad}

Facilita información geo-referencial de pobladores con discapacidad de la parroquia La Matriz del cantón Latacunga. Para el registro, se ha tomado como referencia familias tradicionales latacungueñas, en el que alguno de sus integrantes sea poseedor de alguna discapacidad que dificulte su movilización. El incentivo al registro se propicia en las capacitaciones de evacuación a los moradores de la parroquia ubicada en zona de riesgo, es así como se consigue el registro de ubicación geográfica de 84 moradores de la parroquia mencionada.

Tabla 4

Pobladores con discapacidad de la parroquia La Matriz

\begin{tabular}{|c|c|c|c|c|c|c|}
\hline № & Apellidos & Nombres & Discapacidad & Edad & Latitud & Longitud \\
\hline 1 & Burbano Moreno & María Adriana & mental & 35 & -0.92109 & -78.6213 \\
\hline 2 & Corral Salguero & $\begin{array}{c}\text { Adriana del } \\
\text { Pilar }\end{array}$ & mental & 77 & -0.9306 & -78.6177 \\
\hline 3 & Padilla Hidalgo & Angel María & visual & 82 & -0.937052 & -78.616 \\
\hline 4 & $\begin{array}{c}\text { Reyes de la } \\
\text { Vega }\end{array}$ & $\begin{array}{c}\text { Milton } \\
\text { Plutarco }\end{array}$ & visual & 50 & -0.933909 & -78.6179 \\
\hline 5 & Basante Granja & $\begin{array}{c}\text { Arcesio } \\
\text { Estuardo }\end{array}$ & auditiva & 42 & -0.938852 & -78.6136 \\
\hline .. & .. & .. & .. & .. & .. & .. \\
\hline 80 & $\begin{array}{c}\text { Pumasunta } \\
\text { Quispe }\end{array}$ & $\begin{array}{l}\text { Martha } \\
\text { Cecilia }\end{array}$ & intelectual & 48 & -0.913903 & -78.6324 \\
\hline 81 & $\begin{array}{c}\text { Salazar } \\
\text { Espinosa }\end{array}$ & Gabriel Emilio & lenguaje & 75 & -0.919384 & -78.6213 \\
\hline 82 & Peralvo Barreros & $\begin{array}{c}\text { Irma } \\
\text { Alexandra }\end{array}$ & lenguaje & 65 & -0.923807 & -78.6223 \\
\hline 83 & Carrion Moreno & María Gladis & física & 63 & -0.920318 & -78.6221 \\
\hline
\end{tabular}

En la Tabla 4 se muestra algunos de los campos de varios usuarios que se han registrado en el sistema. Con los datos entregados por parte del usuario se logra también receptar las coordenadas de ubicación de los domicilios de los pobladores con discapacidad de la parroquia en estudio. En la figura 7 se presenta la información geo-referencial de los pobladores con discapacidad que se ha logrado centralizar en la plataforma.

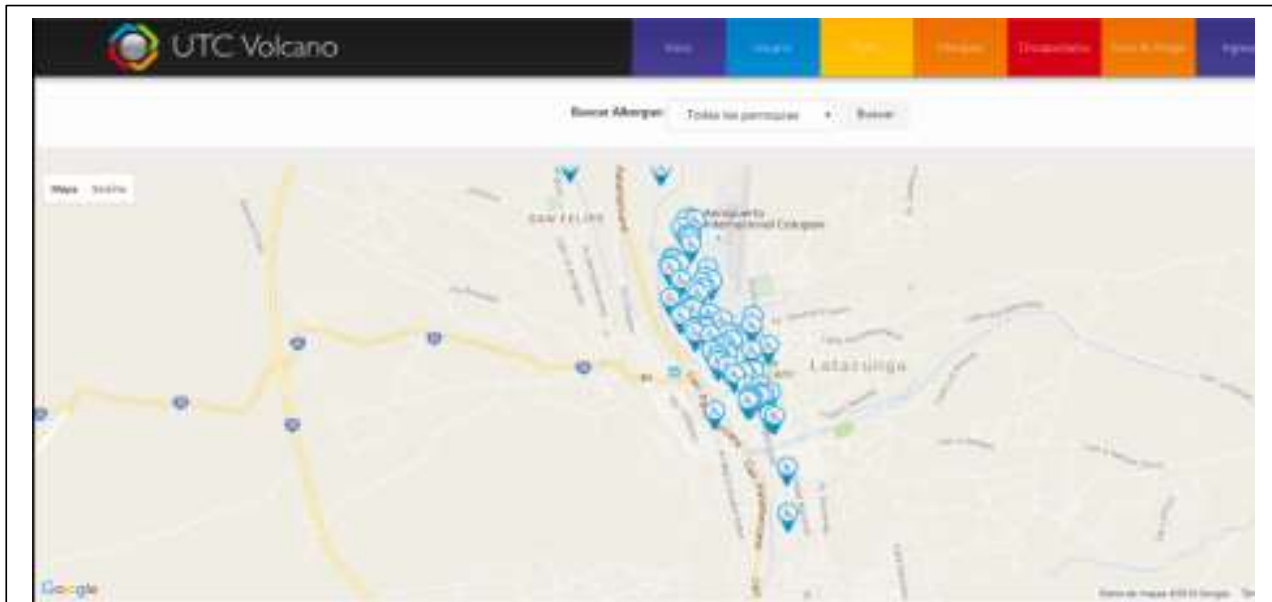

Figura 7 Ubicación geo-referencial en la plataforma web, de los pobladores con discapacidad de la parroquia La Matriz, registrados en la aplicación. 


\section{4) Módulo de Ruta de Evacuación}

Empleando la función de distancia computeDistanceBetween() de la biblioteca de geometría esférica de Google Maps API se calcula la distancia entre la posición geográfica domiciliaria o temporal del usuario en zona de riesgo con la posición geográfica de cada uno de los albergues de la localidad. En efecto se identifica el albergue más cercano y se propone una ruta de evacuación eficiente medida por la distancia del trayecto con menor longitud de recorrido.

En la figura 8 se observa al usuario María Inés Panche Salme en la coordenada geográfica (Lat.=-0.925134, Long.=-78.6199) y al albergue más cercano en la posición geográfica (Lat. $=-0.929485$, Long.=-78.6111) con una distancia de 1085,01 metros de longitud, se describe además la respectiva ruta de evacuación. En consecuencia la Tabla 5 detalla las distancias obtenidas de cada uno de los albergues de la localidad con respecto al usuario. De las evidencias anteriores se destaca que el albergue Coliseo San Sebastián dispone la menor distancia con respecto a los demás.

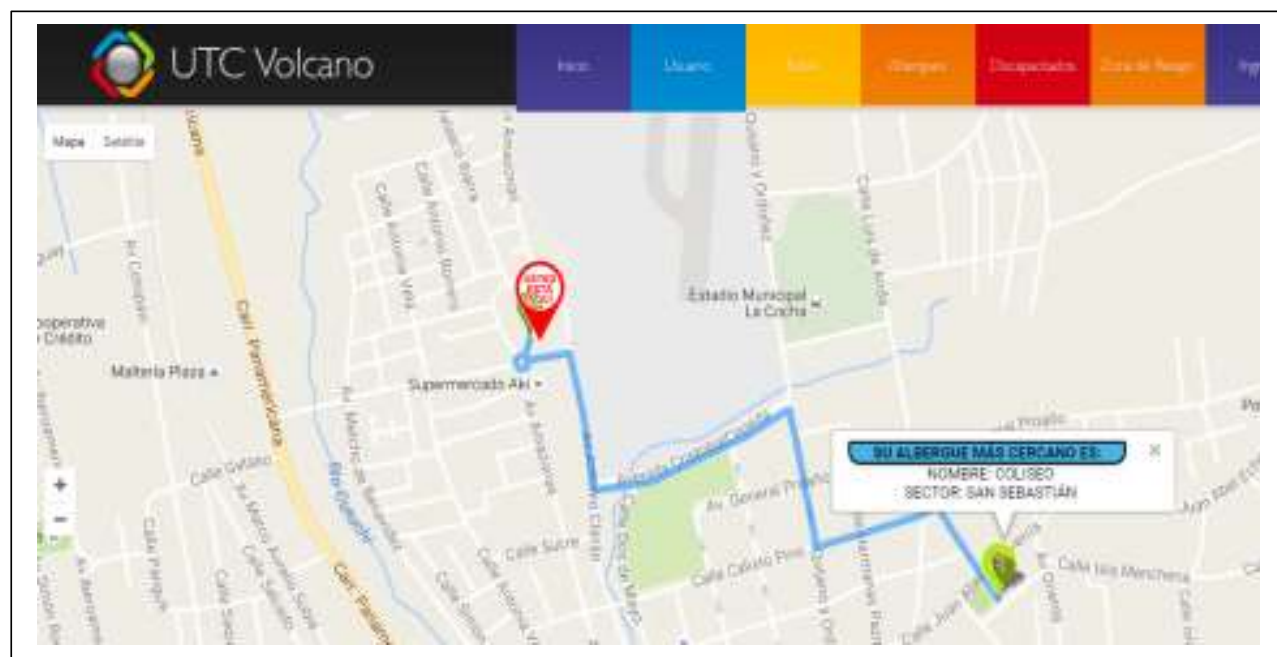

Figura 8 Cálculo del albergue más cercano y la ruta de evacuación a considerar por el usuario para arribar a zona de seguridad.

\section{Tabla 5}

Distancias de los albergues con respecto al usuario

\begin{tabular}{ccc}
\hline Albergue & Ubicación (Lat,Long) & $\begin{array}{c}\text { Distancia } \\
\text { (Metros) }\end{array}$ \\
\hline Coliseo San Sebastián & $-0.929485,-78611177$ & $\mathbf{1 0 8 5 , 0 1}$ \\
\hline Coliseo mayor barrio laguna & $-0.935865,-7860855$ & 1738,66 \\
\hline Casa barrial San Martin Isimbo & $-0.924336,-78603727$ & 1802,32 \\
\hline Iglesia barrio Isimbo 1 & $-0.919997,-78604053$ & 1854,23 \\
\hline $\begin{array}{c}\text { Federacion deportiva de Cotopaxi } \\
\text { barrio Laguna }\end{array}$ & $-0.936114,-78606856$ & 1897,86 \\
\hline $\begin{array}{c}\text { Club femenino Cotopaxi barrio } \\
\text { Isimbo 1 }\end{array}$ & $-0.919778,-78600596$ & 2229,82 \\
\hline
\end{tabular}




\begin{tabular}{ccc}
\hline Casa de la junta de Agua Loma & $-0.903486,-78609362$ & 2680,13 \\
\hline Iglesia barrio Isimbo 2 & $-0.90707,-78601969$ & 2833,17 \\
\hline Iglesia Inchapo & $-0.948919,-7866421$ & 5587,69 \\
\hline Casa comunal Pitigua & $-0.949055,-78664354$ & 5618,98 \\
\hline Iglesia Santa Clara & $-0.781264,-78636323$ & 16119,52 \\
\hline Iglesia Chantillin Grande & $-0.774795,-78.645216$ & 16971,22 \\
\hline.. &.. &.. \\
\hline
\end{tabular}

\section{COMENTARIOS Y CONCLUSIONES}

Se ha implementado una aplicación para registrar y visualizar información de la ubicación geográfica de objetos vinculados al plan de contingencia de la Secretaría Nacional de Gestión de Riesgos del Ecuador. La herramienta es utilizada por pobladores en zona de riesgo de la ciudad de Latacunga, para entregar y recibir información que le ayude al usuario y autoridades de la zona a tomar decisiones de evacuación en caso de una posible erupción del volcán Cotopaxi.

Evidentemente este trabajo requiere de la integración de actores de distintos sectores, su apoyo en cuanto a la vigilancia constante de posible erupción del volcán Cotopaxi, llevaría a una mejor ejecución de planes de evacuación, tal como lo aseveran Stone, Barclay, Simmons, Cole, Loughlin, Ramón, \& Mothes, (2014) con su trabajo "La reducción del riesgo a través de la vigilancia basada en la comunidad: la vigías de Tungurahua, Ecuador", donde establecen una red de actores claves para monitorear el volcán Tungurahua. El sistema propuesto constituiría un complemento excelente para la red establecida por los autores.

Está basada en elementos de información geográfica que conjuntamente con elementos técnicos permite identificar y determinar posiciones (ver figura 6), zonas (ver figura 5), distancias y recorridos geográficos (ver figura 8), de pobladores en zonas de riesgo, albergues y rutas de evacuación.

Ecuador mantiene un plan de divulgación de peligros volcánicos a distintos niveles, y se han publicado temas volcánicos en revistas nacionales y extranjeras, con la intención de concienciar a las autoridades y a la población.

La Secretaría Técnica de Gestión de Riesgos ha realizado campañas puntuales sobre riesgo volcánico y dispone de láminas, trípticos y folletos explicativos sobre las amenazas de un volcán, como protegerse de la ceniza, etc. según exponen (Corominas \& Martí, 2015), evidentemente, contar con sistemas que propicien la información necesaria que ayude a tomar decisiones es una necesidad de primer orden como parte de las estrategias a seguir, el resultado de la presente investigación forma parte de este proceso, como se ha mostrado es un sistema web que servirá para entregar información geográfica de las zonas de riesgo, albergues asignados para el cantón Latacunga, ubicación de personas con discapacidad; la información geográfica de la ubicación domiciliaria del usuario para determinar su mejor ruta de evacuación al albergue más cercano, ante una posible erupción volcánica. 
La presentación de información vinculante al plan de contingencia (ver Tabla 3) de la ciudad de Latacunga a modo de cartografía ha implicado un proceso de administración de elementos de información geográfica, cuya automatización ha sido una tarea compleja.

Sin embargo hay que destacar que el uso de las Tecnologías de la Información y GoogleMaps nos ha permitido, fusionar ubicaciones geográficas y nuevas capas de cartografía. De esta manera se presenta de una forma más iterativa con el usuario, información necesaria para contingencia familiar en el caso de una posible erupción volcánica.

\section{Lista de referencias}

Aguilera, E., \& Toulkeridis, T. (2005). Proyecto de prevención de los riesgos asociados con la erupción del volcán Cotopaxi. Quito, Ecuador: Sotavento.

Andrade, D., Hall, M., Mothes, P., Troncoso, L., Eissen, J., Samaniego, P., . . . Yepes, H. (2005). Los peligros volcánicos asociados con el Cotopaxi. Quito: Corporación Editora Nacional.

Barberi, F., Coltelli, M., Frullani, A., Rosi, M., \& Almeida, E. (1995). "Cronología y características de dispersión de las últimas erupciones tephra del volcán Cotopaxi". Volcanology and Geothermal Research, 217-239.

Chamorro, D., Maisanche, F., \& Puente, D. (14 de Agosto de 2015). "El Instituto Geofísico reportó dos explosiones en el volcán Cotopaxi". El Comercio.

Cornejo, M. (2015). Resolución $N^{a} S G R-042-2015$. Quito, Ecuador: Secretaría de Gestión de Riesgos del Ecuador.

Corominas, O., \& Martí, J. (2015). "Estudio comparativo de los planes de actuacion frente el riesgo volcánico (Costa Rica, El Salvador, Ecuador, España, México, Nicaragua y Chile)". Revista Geológica de América Central, 52.

Del Bosque, I., Fernández, C., Martín-Forero, L., \& Peréz, E. (2012). Los sistemas de información geográfica y la investigación en ciencias humanas y sociales: Confederación Española de Centros de Estudios Locales. Madrid: Confederación Española de Centros de Estudios Locales.

Doocy, S., Daniels, A., Dooling, S., \& Gorokhovich, Y. (2013). "The human impact of volcanoes: a historical review of events 1900-2009 and systematic literature review". PLOS currents, 5 .

Elberg, M., González, L., Hidrobo, F., \& Aguilar, J. (2012). "Análisis numérico del levantamiento de los Andes en Sudamérica con base a energía de deformación". Revista Ciencia e Ingeniería, 43-52.

González-Ferran, O., Munizaga, F., \& Moreno, H. (2015). Síntesis de la evolución volcánica de isla decepción y la erupción de 1970. 
Jara, M. (15 de Agosto de 2015). "Evacúan de forma preventiva Lasso, Mulaló y parte de Latacunga por la actividad del volcán Cotopaxi". El Comercio.

Maisanche, F. (16 de Agosto de 2015). "La normalidad retorno a Latacunga después de la evacuación temporal". El Comercio.

Maisanche, F. (18 de Noviembre de 2015). "Latacunga y Salcedo realizan un simulacro masivo por el volcán Cotopaxi". El Comercio.

Mateos, P. (2013). "Geovisualización de la población: Nuevas tendencias en la web social".

Melo, V., \& Luis, J. (2013). Diseño y Desarrollo de Aplicaciones Interactivas para el Middleware GINGA de Televisión Digital de la Norma ISDB-Tb para brindar Información de los Protocolos de Prevención a la Población en Lugares de Alto Riesgo de Erupciones Volcánicas, Sismos. QUITO/EPN/2013.

Moreno, H. (2015). "La erupción del volcán Mirador en abril-mayo de 1979, lago Ranco-Rininahue, Andes del sur".

Ojeda Zújar, J., Díaz Cuevas, M., Prieto Campos, A., \& Álvarez Francoso, J. (2013). "Línea de costa y sistemas de información geográfica: modelo de datos Para la caracterización y cálculo de indicadores en la costa andaluza".

Olaya, V. (2014). Sistemas de Información Geográfica Tomo I. España: Creative Commons.

Ordoñez, J., Samaniego, P., Mothes, P., \& Schilling, S. (2013). Las potenciales zonas de inundación por lahares en el volcán Cotopaxi. Quito.

Pastor, P., \& Pérez, R. (2008). Evaluación y prevención de riesgos ambientales en Centroamérica. España: Documenta Universitaria.

Pistolesi, M., Cioni, R., Rosi, M., \& Aguilera, E. (2014). "Lahar hazard assessment in the southern drainage system of Cotopaxi volcano, Ecuador: Results from multiscale lahar simulations". Geomorphology, 207, 51-63.

Rodríguez, M., \& Companioni, A. (2014). "Sistema de Información Geográfica para la Industria pesquera". Serie Científica-Universidad de las Ciencias Informáticas, 7, 13-24.

Shroder, J., \& Papale, P. (2014). Volcanic Hazards, Risks and Disasters. Academic Press.

Sinnott, R. (1984). "Virtues of the Haversine". Sky and Telescope.

Stone, J., Barclay, J., Simmons, P., Cole, P., Loughlin, S., Ramón, P., \& Mothes, P. (2014). "Risk reduction through community-based monitoring: the vigías of Tungurahua, Ecuador". Journal of Applied Volcanology, 3.

Sword-Daniels, V., Wilson, T., Sargeant, S., Rossetto, T., Twigg, J., Johnston, D., \& Cole, P. (2014). "Consequences of long-term volcanic activity for essential services in Montserrat: challenges, adaptations and resilience". Geological Society, 39, 471-488. 